\title{
Highly Hydrated Paramagnetic Amorphous Calcium Carbonate Nanoclusters as a Superior MRI Contrast Agent
}

\section{Liang Dong}

University of Science and Technology of China

\section{Yun-Jun Xu}

The First Affiliated Hospital of University of Science and Technology of China

Cong Sui

University of Science and Technology of China

Yang Zhao

University of Science and Technology of China

\section{Li-Bo Mao}

University of Science and Technology of China https://orcid.org/0000-0002-9249-2449

\section{Denis Gebauer}

University of Hannover https://orcid.org/0000-0003-1612-051X

\section{Rose Rosenberg}

University of Konstanz

\section{Jonathan Avaro}

Empa - Swiss Federal Laboratories for Materials Science and Technology

\section{Ya-Dong Wu}

Hefei University of Technology

\section{Huai-Ling Gao}

University of Science and Technology of China https://orcid.org/0000-0001-8908-1967

\section{Zhao Pan}

University of Science and Technology of China

\section{Hui-Qin Wen}

The First Affiliated Hospital of Anhui Medical University

\section{Xu Yan}

Hefei University of Technology

\section{Fei Li}

Hefei University of Technology

\section{Yang Lu}

Hefei University of Technology https://orcid.org/0000-0002-8412-809X 
University of Konstanz https://orcid.org/0000-0002-1148-0308

Shu-Hong Yu ( $D$ shyu@ustc.edu.cn )

University of Science and Technology of China https://orcid.org/0000-0003-3732-1011

\section{Article}

Keywords: amorphous calcium carbonate (ACC), MRI contrast agent, nanoclusters

Posted Date: June 11th, 2021

DOl: https://doi.org/10.21203/rs.3.rs-598198/v1

License: (c) (i) This work is licensed under a Creative Commons Attribution 4.0 International License. Read Full License

Version of Record: A version of this preprint was published at Nature Communications on August 29th, 2022. See the published version at https://doi.org/10.1038/s41467-022-32615-3. 


\title{
Highly Hydrated Paramagnetic Amorphous Calcium
}

\section{Carbonate Nanoclusters as a Superior MRI Contrast Agent}

\author{
Liang Dong ${ }^{1 \star}$, Yun-Jun $\mathrm{Xu}^{3 \star}$, Cong Sui ${ }^{1 \star}$, Yang Zhao ${ }^{1}$, Li-Bo Mao ${ }^{1}$, Denis \\ Gebauer $^{4}$, Rose Rosenberg ${ }^{5}$, Jonathan Avaro ${ }^{6}$, Ya-Dong $\mathrm{Wu}^{2}$, Huai-Ling Gao ${ }^{1}$, \\ Zhao Pan ${ }^{1}$, Hui-Qin Wen ${ }^{7}$ Xu Yan², Fei Li ${ }^{2}$, Yang Lu' ${ }^{2}$, Helmut Cölfen ${ }^{5}$, Shu-Hong \\ $\mathrm{Yu}^{1,2}$
}

${ }^{1}$ Division of Nanomaterials \& Chemistry, Hefei National Laboratory for Physical Sciences at the Microscale, Institute of Energy, Hefei Comprehensive National Science Center, CAS Center for Excellence in Nanoscience, Department of Chemistry, Institute of Biomimetic Materials \& Chemistry, Anhui Engineering Laboratory of Biomimetic Materials, University of Science and Technology of China, Hefei 230026, China.

${ }^{2}$ School of Chemistry and Chemical Engineering, Hefei University of Technology, Hefei, 230009, China.

${ }^{3}$ Department of Radiology, Anhui Provincial Hospital, The First Affiliated Hospital of University of Science and Technology of China, Hefei, 230001, China.

${ }^{4}$ Institute of Inorganic Chemistry, Leibniz Universität Hannover, Callinstr. 9, 30167, Hannover, Germany.

${ }^{5}$ Physical Chemistry, Department of Chemistry, University of Konstanz, Universitätsstr. 10, Konstanz, D-78457, Germany. 
$1{ }^{6}$ Scientist - Center for X-ray Analytics, Empa-Swiss Federal Laboratories for Materials Science and 2 Technology, Lerchenfeldstrasse 5, 9014, St. Gallen, Switzerland.

$3{ }^{7}$ Department of Blood Transfusion, The First Affiliated Hospital of Anhui Medical University, Hefei, 4 230022, China.

$5{ }^{\star}$ These authors contributed equally to this work.

6 Correspondence and requests for materials should be addressed to the author: Yang Lu

7 (yanglu@hfut.edu.cn), Helmut Cölfen (helmut.coelfen@uni-konstanz.de) or Shu-Hong Yu 8 (shyu@ustc.edu.cn). 


\section{Abstract}

2 Amorphous calcium carbonate (ACC) plays a key role as transient precursor in the early stages of biogenic

3 calcium carbonate formation in nature. However, due to its instability in aqueous solution, there is still

4 rare success to utilize ACC in biomedicine. Here, we report the mutual effect between paramagnetic

5 gadolinium ions and $\mathrm{ACC}$, resulting in ultrafine paramagnetic amorphous carbonate nanoclusters (ACNC)

6 in the presence of both gadolinium occluded highly hydrated ACC-like environment and poly(acrylic acid).

7 Gadolinium is confirmed to enhance the water content in ACC, and the high water content of ACNC (23

8 molecules $\mathrm{H}_{2} \mathrm{O}$ per $1 \mathrm{Gd}$ ) contributes to the much enhanced magnetic resonance imaging (MRI) contrast

9 efficiency compared with commercially available gadolinium-based contrast agents. Furthermore, the 10 enhanced $T_{l}$ weighted MRI performance and biocompatibility of ACNC are further evaluated in various

11 animals including rat, rabbit and beagle dog, in combination with promising safety in vivo. Overall, exceptionally facile mass-productive ACNC exhibits superb imaging performance and impressive stability, which provides a promising strategy to design MR contrast agents. 
2 Amorphous calcium carbonate (ACC), which exists widely in nature, plays a key role as transient 3 precursor in the early stages of biomineral formation ${ }^{1-4}$. Using a bioinspired strategy, diverse materials 4 with controlled phase, physical and chemical properties can be achieved ${ }^{5,6}$. The highly hydrated content 5 is a distinctive feature of ACC, and plays a pivotal role in its stabilization ${ }^{6-12}$. As a metastable phase of 6 calcium carbonate, ACC is unstable in aqueous solution and will transform into crystalline phases rapidly 7 owing to dehydration, ion binding and other factors ${ }^{6-8,13}$. Therefore, the potential application of highly hydrated ACC is largely ignored, and there are few successful examples to utilize ACC in biomedicine.

One significant parameter to enhance the contrasting performance of gadolinium-based $T_{l}$ MRI contrast agents is their hydration ${ }^{14}$. With seven non-paired electrons, the gadolinium ion possesses a large magnetic moment and long electron spin relaxation time, leading to numerous clinically available extracellular gadolinium-based contrast agents for $T_{1} \mathrm{MRI}^{14,15}$. In virtue of versatile functionalization to interact with biomolecules in vivo and lower gadolinium ion leakage rates benefitting from an inorganic nanostructure, Gd-based inorganic nanoagents attracted considerable attention ${ }^{15,16}$. Unfortunately, the hydration of gadolinium-based nanoparticles suffers from high-temperature synthesis, though the consequent ion leakage is minimized by the confined nanostructure compared with chelate complexes ${ }^{15}$.

Herein, we first introduce gadolinium ions into the ACC mineralization process, which have been proven to be integrated into the final amorphous calcium carbonate phase. In this amorphous system, gadolinium ions in conjunction with poly(acrylic acid) facilitated enhanced hydration water and stability of nanoclusters, while the confinement of gadolinium ions by carbonate improved the biocompatibility and performance, indicating the resulting product has noteworthy MRI contrast agent properties. Furthermore, a mutual effect between the paramagnetic lanthanide gadolinium ion and amorphous 
calcium carbonate is discovered, which contributes to the maximized hydrated content in the as-prepared amorphous composite nanoclusters and an extremely high longitudinal relaxation. The final amorphous nanoclusters possessed an extremely high water to $\mathrm{Ca}$ ratio (water/Ca $=7.2$ ) compared with the normal ACCs (ratios remained constant at about $0.4-1.9)$. The longitudinal relaxivity of ACNC $(37.93 \pm 0.63$ $\mathrm{mM}^{-1} \cdot \mathrm{s}^{-1}$ under 3.0 T) has also benefited from the high water content, which is ten times higher than that of the commercially available MR contrast agent gadopentetic acid (Gd-DTPA) and highly resistant to ion leakage so it can serve as a potential MR contrast agent.

\section{Results}

Characterization of ACNC. So far, besides different kinds of organic additives such as biomolecules and polymers, magnesium is the only inorganic cation that has been reported capable of stabilizing $\mathrm{ACC}^{6,17}$. The gadolinium ion, whose ionic radius is closer to that of the calcium ion than that of the magnesium, can be regarded as a smaller calcium ion analogue with higher valence and hydration energy ${ }^{18,19}$. Similarly to the chelation between poly(acrylic acid) (PAA) and calcium for the enhanced stability of $\mathrm{ACC}^{7,20}$, carboxylate groups in PAA have the potential to chelate gadolinium ions, allowing the bound complex to later be solidified into carbonate ${ }^{21}$.

As shown in Fig. 1A, calcium chloride and gadolinium chloride were first mixed with PAA, and equimolar sodium carbonate solution was then added under robust stirring. This efficient synthesis at room temperature proved to yield amorphous carbonate nanoclusters (ACNC) in the presence of both gadolinium and PAA. Two liters of aqueous product can be facilely produced, indicating the scalability and reproducibility of the method reported here (Fig. 1B). The presence of clusters dispersed in normal saline was reflected by the Tyndall effect (Fig. 1C). As shown in Fig. 1D, the spherical morphology was further investigated by cryo-TEM, and proved to be comparable with ACC as reported previously ${ }^{22}$. The 
1 nanoclusters observed by STEM (fig. S1) were in accordance with HAADF-STEM observations and the 2 amorphous character of clusters was validated by SAED analysis (Fig. 1E). EDS data showed the uniform 3 distributions of gadolinium and calcium in the cluster aggregates (fig. S2, A and B).

ACNC exhibited no crystalline peak in the XRD pattern, even after being dispersed in aqueous solution 5 for 6 months (fig. S3). The ACNC was further studied by X-ray Absorption Spectroscopy (XAS) and extended X-ray absorption fine structure (EXAFS) analysis, and the Ca-O short-range environment within ACNC was revealed to closely relate to those in the ACC standard and previously reported ACCs ${ }^{10,11,23}$ (Fig. 1, F and G, tables $\mathrm{S} 1$ and $\mathrm{S} 2$ ). The size distribution of ACNC received from SAXS fitting analysis showed an average radius of $1.3 \mathrm{~nm}$ (Fig. 1, H and I). In addition, the particle size of ACNC was measured by dynamic laser scattering to be approximately $7.46 \pm 1.30 \mathrm{~nm}$ which is higher than the diameter from SAXS of $2.6 \mathrm{~nm}$ due to intensity weighing (fig. S4).

Thermogravimetric analysis (TGA) and differential scanning calorimeter (DSC) results for ACNC showed a loss over $20 \mathrm{wt} \%$ upon heating to $300{ }^{\circ} \mathrm{C}$ due to the dehydration of the ACC, indicating a high water content of ACNC. In addition, the pyrolysis of PAA occurred between 300 and $500{ }^{\circ} \mathrm{C}$, and carbonate was decomposed above $550{ }^{\circ} \mathrm{C}$ (Fig. 1J and fig. S5). Based on the TGA result, the composition of ACNC included approximately $20 \%$ water content, $40 \%$ PAA and $40 \%$ amorphous carbonate. The absorbance observed in TGA coupled FTIR (TG-FTIR) on ACNC at 2358 and $2322 \mathrm{~cm}^{-1}$ above $550{ }^{\circ} \mathrm{C}$ corresponded to the asymmetric stretching vibration of $\mathrm{CO}_{2}$ from the decomposition of carbonate (Fig. $1 \mathrm{~K}$ ). As shown in fig. S6, the split band at 1415 and $1454 \mathrm{~cm}^{-1}$ in Fourier transform infrared spectroscopy (FTIR) can be assigned to the asymmetric stretch vibration of the carbonate ions in typical ACC environments ${ }^{19}$. A broad peak centered at $1086 \mathrm{~cm}^{-1}$ attributed to the internal $\mathrm{CO}_{3}{ }^{2-}$ symmetric stretch was further confirmed by Raman spectroscopy (fig. S7). 
1 Highly hydrated content of ACNC. In order to investigate the contribution of each component to the 2 highly hydrated content and ACC-like environment, a series of control samples with varying composition were synthesized as listed in table S3, including a Gd-occluded ACC composite (ACC-Gd), PAAstabilized ACC (ACC-PAA), amorphous gadolinium carbonate (AGC), PAA-stabilized AGC (AGC-PAA) and two carbonate-free chelates (PAA-Ca and PAA-Ca/Gd). XRD patterns and Raman spectra were performed to identify the amorphous phase (fig. S8, A and B). In the absence of PAA, ACC-Gd showed a typical morphology for aggregated ACC nanoparticles with a uniform distribution of Gd and Ca (fig. S9, A to D). The extended X-ray absorption fine structure (EXAFS) results further confirmed the presence of ACC-like environments (fig. S10, A and B).

Attractively, there was still a higher water content held in ACC-Gd as compared to pure ACC even in deionized water for a long time. The weight loss before $300{ }^{\circ} \mathrm{C}$ of ACC-Gd and ACC powder exposed to the air over 6 months was more than $10 \%$ and only $0.1 \%$, respectively (fig. S11). In both TGA and volumetric Karl Fischer titration measurement results, the weight loss before $300{ }^{\circ} \mathrm{C}$ could be assigned to the loss of water, and ACNC exhibited the highest water content of calculative $20 \mathrm{wt} .-\%$ among the products with different composition (Fig. 2A). According to previous reports, the water contents per mole of calcium in additive-free ACC and PAA-stabilized ACC were typically located in the range of 0.4-1.58 and 1.33-1.93, respectively ${ }^{20,24}$. In contrary, both ACC-Gd and ACC-PAA showed a higher water to Ca ratio, and the highest ratio was obtained in $\mathrm{ACNC}$ (water/Ca = 7.2) (Fig. 2B), indicating a synergetic enhancement of water binding by PAA and Gd in these amorphous nanoparticles. More importantly, to compare the ratio of water to $\mathrm{Gd}$ as listed in Fig. $2 \mathrm{C}$, the hydrated content per mole gadolinium in the presence of both ACC-like environment and PAA in ACNC is more than four times that of PAA-occluded gadolinium carbonate (PAA-AGC). Compared with AGC, the presence of ACC in the Gd-ACC composite 
1 also resulted in a two-fold increase of the hydrated content per gadolinium ion.

To investigate the origin of the hydration, analytical ultracentrifugation (AUC) was applied to determine the hydration water of ACNC via the frictional ratio and Perrin function assuming a spherical shape seen in the electron microscopy images (table S4). The samples show sedimentation coefficients in the order of $10^{-13} \mathrm{~s}$, which is typical for prenucleation clusters (PNCs) that have similar sizes ${ }^{25}$. The ACNCs have a diameter of $1.5 \mathrm{~nm}$, which is even smaller than the one found by SAXS, though still in reasonable agreement. Their molar mass of $2200 \mathrm{~g} / \mathrm{mol}$ indicates that they consist of approximately $20 \mathrm{CaCO}_{3}$ ion 8 pairs including the $\mathrm{Gd}^{3+}$ ions. The determined amount of bound hydration water in solution of 8.8 mol

$\mathrm{H}_{2} \mathrm{O}$ per $\mathrm{CaCO}_{3}$ (and 23.0 mol $\mathrm{H}_{2} \mathrm{O}$ per $\mathrm{Gd}^{3+}$ ), is considerably higher than in common ACCs (Fig. 2, B and C, table S5). Notably, the water content of ACNC determined by AUC is in reasonable agreement with the results from TGA, considering that the hydration was determined in wet and dry states, respectively ${ }^{6}$.

Stable high relaxivity of ACNC. Besides the contribution of the dopant of the gadolinium ion to the highly hydrated ACC content, a typical paramagnetic behavior was achieved in the amorphous carbonate nanoclusters as shown in the M-H curve (Fig. 3A). Both the high hydration and confinement of the gadolinium ions, the paramagnetic ACNC was conducive to increasing MRI contrast performance. The $T_{1}$ relaxation times of $\mathrm{ACNC}$ dispersed in normal saline at varying concentrations were measured using a 3.0 Tesla MR scanner. As shown in the $T_{1}$ map (Fig. 3B), the longitudinal relaxivity $\left(r_{1}\right)$ of ACNC reached $37.21 \mathrm{mM}^{-1} \cdot \mathrm{s}^{-1}$ (Fig. 3C), which was ten times higher than that of commercially used Gd-DTPA (3.19 $\mathrm{mM}^{-}$ $\left.{ }^{1} \cdot \mathrm{s}^{-1}\right)$. Besides the contribution from the large size of macromolecules and nanoparticles, ACNC possessed four times higher longitudinal relaxivity compared to PAA modified amorphous gadolinium carbonate (AGC-PAA) (7.91 $\left.\mathrm{mM}^{-1} \cdot \mathrm{s}^{-1}\right)$, which was attributed to the high hydration content of ACC-like 
1 environments. This high content of water may be primarily attributed to the stronger hydration ability of 2 trivalent $\mathrm{Gd}^{3+}$ as compared to divalent $\mathrm{Ca}^{2+}$ ions ${ }^{19}$, which reasonably enhanced inner-sphere and outer3 sphere relaxations of the ACC like environment occluded $\mathrm{ACNC}^{14}$. After three tests, the $r_{1}$ value of ACNC 4 was calculated to be approximately $37.93 \pm 0.63 \mathrm{mM}^{-1} \cdot \mathrm{s}^{-1}$ (Fig. 3D).

In addition, $r_{1}$ and $r_{2}$ values of $\mathrm{ACNC}$ were measured under different magnetic fields $(3.0 \mathrm{~T}$ and $0.5 \mathrm{~T})$, and its corresponding $r_{2} / r_{1}$ ratio was calculated (Fig. 3, E and F). The $r_{1}$ value of ACNC measured on 3.0 $\mathrm{T}$ was as high as $38.19 \mathrm{mM}^{-1} \mathrm{~s}^{-1}$, and the corresponding $r_{2}$ value and $r_{2} / r_{1}$ ratio were $72.49 \mathrm{mM}^{-1} \mathrm{~s}^{-1}$ and 1.90, respectively. Using a low field MR scanner system $(0.5 \mathrm{~T})$, the corresponding $r_{1}$ and $r_{2}$ values of ACNC were $66.37 \mathrm{mM}^{-1} \mathrm{~s}^{-1}$ and $78.04 \mathrm{mM}^{-1} \mathrm{~s}^{-1}$, respectively, and the $r_{2} / r_{1}$ ratio was 1.18 .

High stability is essential for gadolinium-based contrast agents, as transmetallation will lead to the release of dissociated gadolinium ion with reported toxicity such as nephrogenic systemic fibrosis ${ }^{14,26}$. Laurent and Muller reported the poor kinetic inertness against transmetallation for linear gadoliniumbased contrast agents such as commercially used Gd-DTPA ${ }^{27}$. As shown in Fig. 3G, the PAA-Ca/Gd chelate exhibited an even worse inertness than Gd-DTPA after exposure to $\mathrm{Zn}^{2+}$ in PBS for $48 \mathrm{~h}$. On the contrary, ACNC demonstrably enhanced the stability against transmetallation, due to the gadolinium confinement in ACC-like environments.

Stability and biocompability of ACNC. The absorption spectra of arsenazo III is generally used to detect the leakage of gadolinium ion from Gd-based nanocomposite ${ }^{28,29}$. When the Arsenazo III aqueous solution was mixed with $\mathrm{Gd}^{3+}$, pink solution turned blue due to the formation of arsenazo-Gd ${ }^{3+}$ complex (Fig. 4A). As shown in Fig. 4B, free gadolinium ion at a low concentration of $1 \mu \mathrm{g} / \mathrm{mL}$ was detectable by arsenazo III mediated absorption spectra. However, in the normal saline dispersion of ACNC, no leakage of free gadolinium ions in one week dialysis was detected using this colorimetric analysis, which was further 
1 confirmed by ICP-MS (Fig. 4C). In sharp contrast to ACNC, PAA-Ca/Gd chelate showed an obvious

2 leakage compared with ACNC, further confirming the confinement of gadolinium ions by carbonate.

Moreover, ACNC was dispersed in human serum at $1 \mathrm{mmol}(\mathrm{Gd}) / \mathrm{L}$. Meanwhile, to simulate the

elevated phosphate concentrations in serum in patients with end-stage renal disease ${ }^{30}$, the same concentration of ACNC was dispersed in human serum supplemented an additional phosphate concentration of $10 \mathrm{mmol} / \mathrm{L}$ for 15 days, followed by dialysis at different time points. No leakage of free gadolinium ions was detected in dialysates using ICP-MS and colorimetric analysis (Fig. 4, D and E, fig. $\mathrm{S} 12)$, indicating the low dissociation risk for $\mathrm{ACNC}$ in the retention period in vivo.

To determine whether ACNC cause hemolysis, ACNC at different concentrations were incubated with haemocylolysis even at a high concentration of $0.5 \mathrm{mg}(\mathrm{Gd}) / \mathrm{mL}$, suggesting a good blood compatibility

(Fig. 4F). MTT assay was used as an evaluation of cytotoxicity by co-culturing human umbilical vein endothelial cells (HUVEC) with ACNC for $24 \mathrm{~h}$. No obvious reduction of viability in cells was induced after exposure to ACNC, even at a high concentration of $0.5 \mathrm{mg}(\mathrm{Gd}) / \mathrm{mL}$ (fig. S13). Encouraged by the results thus far, a series of subcellular compatibility studies were carried out using human renal tubular epithelial cell (HK-2). Healthy mitochondria of HK-2 cells were demonstrated by mitochondrial membrane potentials (MMP) study (fig. S14). The immunofluorescent TdT-mediated dUTP Nick-End Labeling (TUNEL) staining assay of HK-2 showed ACNC had no accumulated damage to the nuclear DNA of cells (fig. S15). An EdU (5-Ethynyl-2'-deoxyuridine) assay was performed and confirmed that ACNC had no effects on cell proliferation (fig. S16).

Meanwhile, histological sections of kidney and other major organs were collected 2 weeks after the intravenous injection in mouse at a dose of $5 \mathrm{mg} \mathrm{Gd} / \mathrm{kg}$, and no evidence of pathologic change was 
1 identified in H\&E stained images (fig. S17, and fig. S18). 3 days and 3 weeks after intravenous injection 2 of ACNC in mouse, all hematologic and biochemical parameter values were in accordance with the standard range and our control groups (fig. S19). To further test in the large animal beagle dog at a high dosage ( $9 \mathrm{mg} \mathrm{Gd} / \mathrm{kg} \mathrm{bw}$ ), there was no obvious difference of hematologic and biochemical parameters between control and experimental groups after intravenous injection (fig. S20), suggesting that physiological functions were not impaired by ACNC.

In vivo MR imaging and metabolism of ACNC. The enhancement of $T_{l}$ weighted MRI performance of ACNC was further confirmed by MR angiography in rat, rabbit and beagle dog. After the bolus intravenous injection of $\mathrm{ACNC}$ at a low dose ( $3 \mathrm{mg} / \mathrm{kg}$ bodyweight), jugular, carotid, aorta and caudal vena cava can be clearly identified (Fig. 5, A to C), and the dosage is less than 1/5 of the typically employed value in clinic. The whole-body angiography images of rat, rabbit and upper body of beagle dog exhibited better angiographic contrast and more details in comparison with that of the Gd-DTPA control group. As shown in Fig. 5D, the contrast enhancement of ACNC in vivo was further quantitatively reflected by the mean value of signal-noise ratio (SNR) on rabbit and beagle dog, respectively ${ }^{31}$.

Recently, clinical translation of nanosized biomaterials attracted great attention, promising the development of novel and specific nanomedical tools for diagnosis and therapy ${ }^{16,32-35}$. To avoid the potential risk in vivo, renal clearance is the desirable and preferred excretion route for medical agents for minimal catabolism and body exposure. However, compared to small molecular contrast agents, nanosized MR contrast agents, especially the FDA approved nanosized iron oxide injections suffer from their poor renal excretion as a result of their large size distribution $(>20 \mathrm{~nm})^{36}$. Besides the conventional elimination from liver (fig. S21), the quick renal clearance of ACNC from blood vessels was observed in the MRA images after intravenous injection (fig. S22, A and B). Moreover, the bladder of beagle dog was also 
1 brightened within 20 minutes in the $T_{l w}$ image (fig. S23). In addition, as shown in the blood concentration-

2 time curve in mouse and beagle dog measured by ICP-AES, it could be effectively cleared from blood 3 vessels in 6 hours and there is rarely any residual content of gadolinium after $24 \mathrm{~h}$ (Fig. 6, A and B). In 4 the collected rat urine after the intravenous injection of ACNC, the content of gadolinium was detected by 5 ICP-AES and demonstrated a renal clearance efficiency of approximately $13 \%$ ID at $24 \mathrm{~h}$ (fig. S24), which 6 is comparable to that of gold nanoclusters with similar diameters ${ }^{37}$. Abundant SAED amorphous cluster 7 aggregates could be observed in TEM images of dialyzed urine (Fig. 6C), and EDS mapping revealed a 8 matching distribution of gadolinium, calcium, carbon and oxide elements in these aggregates 9 corresponding to ACNC (Fig. 6, D and E). Physicochemical and physiological stability in synergy with low injection dosage and partial clearance via kidney led to in vivo biocompatibility and potential translational ability of these gadolinium-based amorphous carbonate clusters.

\section{Discussion}

A number of gadolinium-based contrast agents have been designed and approved worldwide for $T_{1}$ MR imaging $^{14}$. As a result of a high density of free electrons in the valence band and versatile functionalization to interact with biomolecules in vivo, inorganic nanomaterials serving as contrast agents in various imaging modalities are anticipated to achieve the clinical translation of nanomedicine ${ }^{15,38}$. Therefore, the scientists studied indefatigably including diverse fabrication methods and clearing pharmacokinetics in order to investigate the translatability of Gd-based inorganic nanoparticles ${ }^{16}$. In consideration of the hazard originated from the release of free Gd ions, a specific aim was to reduce the dose of injected nanoparticles via the enhanced performance.

The hydration level plays a significant role in determining the contrasting performance of a MR contrast agent $^{14}$. Unfortunately, the hydration content of Gd-based nanocrystals is limited by traditional high 
1 temperature synthesis processes ${ }^{15}$. Worth mentioning is that the high moisture content, including interior

2 water and deeply located structural water is the most distinctive feature of $\mathrm{ACC}^{7-11}$. However, the potential

3 application of unstable hydrated ACC is largely ignored. Interestingly, the ionic radius of the gadolinium

4 ion is very close to that of the calcium ion, implying a possible interaction between gadolinium ions and

5 calcium ions that can be exploited by us.

6 In summary, our study confirms mutual effects between the paramagnetic lanthanide gadolinium ion

7 and amorphous calcium carbonate, which is beneficial to maximizing the water content in the obtained

8 amorphous composite nanoclusters. The material is synthesized through a facile one-pot process at room

9 temperature, enabling the large scale and cost effective production. Importantly, this high water content

10 contributes to the transparent MRI contrasting enhancement of gadolinium-based nanoclusters. In

11 combination with their low toxicity, partial renal clearance and easy potential for mass production, our

12 work enables further identification of the biomedical potential of ACC composites, and we anticipate that

13 it could lead to a new generation of more efficient diagnostic agents on the basis of amorphous

14 nanoclusters in the future. 


\section{Methods}

Materials. Anhydrous calcium chloride, anhydrous sodium carbonate and ethyl alcohol were purchased from Sinopharm Chemical Reagent Co. Ltd (Shanghai). Gadolinium chloride hexahydrate $\left(\mathrm{GdCl}_{3} \cdot 6 \mathrm{H}_{2} \mathrm{O}\right.$, 99.95\%) were purchased from Yutai QingDa Fine Chemical Co., Ltd. (Shandong, China). Poly(acrylic acid) (PAA, $\mathrm{Mw} \approx 1800$ ) was purchased from Aldrich. All reagents were used as received without further purification. The commercially used Gd-DTPA was produced by Guangdong Consun Pharmaceutical Group, China (specification: $20 \mathrm{~mL}$ ).

Sample preparation. In a typical procedure to synthesize additive-free amorphous calcium carbonate (ACC), $10 \mathrm{~mL} \mathrm{Na} \mathrm{CO}_{3}$ aqueous solution $\left(0.1 \mathrm{~mol} \cdot \mathrm{L}^{-1}, 10 \mathrm{~mL}\right)$ was poured into calcium chloride $(0.1$ $\mathrm{mol} \cdot \mathrm{L}^{-1}, 10 \mathrm{~mL}$ ) aqueous solutions under vigorous magnetic stirring for 15 seconds. The aqueous suspension after adding $20 \mathrm{~mL}$ ethanol were centrifuged at $3000 \mathrm{rpm}$ for $2 \mathrm{~min}$ immediately, then the precipitates were washed by ethanol, centrifuged for $5 \mathrm{~min}$ at $8000 \mathrm{rpm}$ twice.

In a typical procedure to synthesize $\mathrm{ACNC}$, anhydrous calcium chloride $\left(\mathrm{CaCl}_{2}, 0.1 \mathrm{~mol} \cdot \mathrm{L}^{-1}\right)$, gadolinium chloride hexahydrate $\left(\mathrm{GdCl}_{3}, 0.02 \mathrm{~mol} \cdot \mathrm{L}^{-1}\right)$ and PAA $(0.45 \mathrm{~g})$ were dissolved in $10 \mathrm{~mL}$ DIW with magnetic stirring. Anhydrous sodium carbonate $\left(\mathrm{Na}_{2} \mathrm{CO}_{3}, 0.1 \mathrm{~mol} \cdot \mathrm{L}^{-1}, 10 \mathrm{~mL}\right)$ was poured into the above solution with vigorous magnetic stirring for $1 \mathrm{~min} .20 \mathrm{~mL}$ of ethanol was added to terminate the reaction. The mixture was immediately centrifuged for $2 \mathrm{~min}$ at $3000 \mathrm{rpm}$, and precipitates were resuspended by DIW, centrifuged for $5 \mathrm{~min}$ at $8000 \mathrm{rpm}$ twice. For liter-scale synthesis, the reaction volume was scaled up 50 times and proceeded under robust mechanical agitation.

For the synthesis of ACC stabilized by PAA (ACC-PAA), $0.45 \mathrm{~g}$ PAA was used in the synthesis process without the addition of gadolinium salt, and the product was washed by ethanol. For the preparation of 
1 ACC-Gd, PAA was absent in the synthesis process and anhydrous $\mathrm{CaCl}_{2}\left(0.1 \mathrm{~mol} \cdot \mathrm{L}^{-1}\right)$ and $\mathrm{GdCl}_{3}(0.02$ $2 \mathrm{~mol} \cdot \mathrm{L}^{-1}$ ) were mixed in $10 \mathrm{~mL}$ DIW. Then the obtained ACC-Gd was washed by ethanol.

In a typical procedure to synthesize amorphous gadolinium carbonate (AGC) and AGC-PAA, $\mathrm{Na}_{2} \mathrm{CO}_{3}$

$4\left(0.1 \mathrm{~mol} \cdot \mathrm{L}^{-1}, 10 \mathrm{~mL}\right)$ was poured into $10 \mathrm{~mL} \mathrm{GdCl}$ aqueous solution $\left(0.067 \mathrm{~mol} \cdot \mathrm{L}^{-1}, \mathrm{~mol}\left[\mathrm{GdCl}_{3}\right]\right.$ : $5 \operatorname{mol}\left[\mathrm{Na}_{2} \mathrm{CO}_{3}\right]=2: 3$ ) under vigorous magnetic stirring for 15 seconds, then $20 \mathrm{~mL}$ ethanol was poured 6 into the aqueous suspension. After centrifuged at $3000 \mathrm{rpm}$ for $2 \mathrm{~min}$ immediately, the precipitates were 7 washed by ethanol, centrifuged for $5 \mathrm{~min}$ at $8000 \mathrm{rpm}$ twice. In addition, in the presence of $0.45 \mathrm{~g}$ PAA 8 in the $\mathrm{GdCl}_{3}$ aqueous solution, AGC-PAA was prepared in the same procedure.

9 Characterization. Transmission electron microscopy (TEM) were carried out on H7700 (Hitachi, Japan) performed on Tecnai F20 Transmission Cryo-Electron Microscopy. High-angle annular dark field scanning TEM (HAADF-STEM), energy dispersive spectrometer (EDS) and EDS mapping were performed on a field emission high resolution transmission electron microscope (FEI, Talos F200X). The hydrodynamic diameter was calculated on a Nano ZS-90 Zetasizer (Malvern Instruments Corp, UK). Xray powder diffraction (XRD) patterns were performed on a rotating anode X-ray diffractometer 
1 device (SQUID) magnetometer (Quantum Design SQUID-VSM). ICP-AES were performed on an Optima

27300 DV instrument. UV-vis spectra were measured on a Shimadzu UV-2600 spectrophotometer at room

3 temperature.

4

Small-angle X-ray scattering (SAXS). Small-angle X-ray scattering (SAXS) analysis were performed on a SAXSpoint 2.0 (Anton Paar). The size distribution function as determined by SAXS is evaluated using GIFT (Generalized indirect Fourier transformation) software with precondition of homogeneous spheres weighted by number. It is calculated using the following formulas ${ }^{41}$ :

$$
I(q)=\int_{0}^{R_{\max }} R^{6} \cdot D_{N}(R) \cdot\left(3 \cdot \frac{\sin (q R)-q R \cos (q R)}{(q R)^{3}}\right)^{2} \cdot d r
$$

EXAFS sample preparation, measurement and data fitting. X-ray Absorption Spectroscopy

(XAS) measurements at the calcium K-edge $(4.0381 \mathrm{keV})$ were undertaken at the Elettra synchrotron Italy, operating at an energy of $2 \mathrm{GeV}$ and current of $300 \mathrm{~mA}$. Samples were ground in an agate mortar, diluted with graphene powder and compacted into thin pellets. An optimal sample thickness of $\sim 300 \mu \mathrm{m}$ and optimal calcium concentration after optimal dilution with graphene powder was prepared for each sample including ACNC, ACC-Gd and ACC standards. Three full scans per sample of the X-ray absorption near edge structure (XANES) and extended X-ray absorption fine structure (EXAFS) were collected in transmission mode using a FMB-OXFORD ion chamber detector, in steps of $5 \mathrm{eV}$ in the pre-edge region (from $3738.43 \mathrm{eV}$ to $4028.53 \mathrm{eV}$ ) and $0.2 \mathrm{eV}$ in the edge region (from $4061.51 \mathrm{eV}$ to $4061.71 \mathrm{eV}$ ), gradually increasing to $2.6 \mathrm{eV}$ in the post-edge region (4061.71 eV to $4589.30 \mathrm{eV}$ ) up to $\mathrm{k}$ $=13$. Alignment, energy calibration and deglitching were performed using built-in features of the Athena software package ${ }^{42}$. The first coordination shell around calcium $(\mathrm{Ca}-\mathrm{O})$ was fitted by generating a single scattering pathway of Ca-O based on crystallographic data of calcite. Analysis of single scattering pathways was undertaken from 1.3 to $2.53 \AA$ in $\mathrm{R}$ space on Fourier transformed $\mathrm{k}^{2}$-weitghed 
1 data from 3 to $9.1 \AA$ A. Standards errors associated with the EXAFS data fitting over the k-range used here

2 are $15 \%$ for the first-shell coordination number, $0.03 \AA$ for the radial distance and $15 \%$ for the Debye-

3 Waller factors.

4

Independent data points were determined by Stern's rule defining the fundamental limitation to the amount of information that can be determined by XAFS. According to Stern's rule, the number of parameters $\left(\mathrm{N}_{\mathrm{par}}\right)$ used for the fit should be strictly inferior to the number of independent $\left(\mathrm{N}_{\text {ind }}\right)$ parameters defined as $2 \Delta \mathrm{k} \Delta \mathrm{R} / \pi+2$, where $\Delta k$ and $\Delta R$ are the fitted range in $k$ and $R$ space, respectively ${ }^{43,44}$. If $\mathrm{N}_{\text {ind }}<$ $\mathrm{N}_{\text {par }}$, the model cannot be taken as a proof of the coordination environment because the data set is underdetermined by the level of complexity of the model. In this case, $\mathrm{N}_{\mathrm{ind}}=7.5$ for ACC standard, ACCGd and ACNC sample, $\mathrm{N}_{\text {ind }}=8.16$ for ACC-PAA and $\mathrm{N}_{\text {par }}=4$ as such than $\mathrm{N}_{\text {ind }}>\mathrm{N}_{\text {par. }}$ The multicomponent model yielding the best fit of the experimental data is considered as reasonable and can thus be considered as a likely representation to characterize the calcium coordination environment. Linear combination was performed on the XANES and near-EXAFS region (from $30 \AA^{-1}$ before to $80 \AA^{-1}$ after edge jump). Samples ACC-Gd, ACNC and PAA-Gd/Ca were compared to standards samples ACC-PAA and PAA-Ca.

Analytical ultracentrifugation (AUC). The AUC measurements were performed on a modified Optima XL-A (Beckman Coulter, Palo Alto, CA, United States) using an absorbance optics and an advanced Rayleigh interference optics developed by Nanolytics ${ }^{45}$. 12-mm path length double-sector titanium centerpieces with saphire windows (Nanolytics, Potsdam, Germany) were used for all experiments. For ACNC sample measurement, the original ACNC sample was pre-sedimented on a UNIVERSAL 320 Hettich centrifuge (Hettich, Tuttlingen, Germany) for $20 \mathrm{~min}$ at $9000 \mathrm{rpm}$. $360 \mu \mathrm{L}$ of pretreated ACNC solution was used in the sample sector and $360 \mu \mathrm{L}$ of a 1:175 diluted (with $0.154 \mathrm{M} \mathrm{NaCl}$ ) pre-reaction 
1 PAA-Ca/Gd solution (aqueous mixture including PAA, calcium chloride and gadolinium chloride) in the 2 reference sector. For PAA sample measurement as reference, $10 \mathrm{mg}$ PAA (1800 Da) were dissolved in 1 $3 \mathrm{~mL}$ of $0.154 \mathrm{M} \mathrm{NaCl}$ solution. All samples were investigated at $20^{\circ} \mathrm{C}$ and $60000 \mathrm{rpm}$, corresponding to 4 a centrifugal force up to $290000 \mathrm{~g}$.

Sedimentation velocity data were evaluated with Sedfit and UltraScanIII. In Sedfit (version 16.1c by Peter Schuck $\left.{ }^{46}\right)$, the $1 \mathrm{~s}-\mathrm{g}^{*}(\mathrm{~s})$ and continuous $\mathrm{c}\left(\mathrm{s}, \mathrm{ff}_{0}\right)$ models were used. For calculation of the sedimentation coefficient distributions $g(s)$ with the least-squares-g*(s) $\operatorname{model}^{47}$, data were fitted with a Tikhonov-Phillips regularization using a confidence level (F-ratio) of 0.683 and a resolution of 100 grid points. For determination of the sedimenting particle density ${ }^{48}$ with the $\mathrm{c}\left(\mathrm{s}, \mathrm{ff}_{0}\right)$ analysis ${ }^{49}$, data were fitted with a maximum entropy regularization using a confidence level (F-ratio) of 0.683 and a resolution of 100 grid points in s and 10-20 grid points in $\mathrm{f} / \mathrm{f}_{0}$. The $2 \mathrm{D} \mathrm{c}\left(\mathrm{s}, \mathrm{ff}_{0}\right)$ distributions were plotted using MATLAB software version R2017b, 64 bit from MathWorks. For calculation of the sample density from 2D distributions, a MATLAB mask script developed by Quy Khac Ong ${ }^{50}$ was used. For characterization of anisotropy, UltraScanIII ${ }^{51}$ (version 4.0, revision 5699) was used for performing the two-dimensional spectrum analysis (2DSA) ${ }^{52}$. The 2DSA-Monte Carlo (MC) analyses were performed with 50 iterations on a supercomputer.

The function defining the shape, flexibility and degree of solvation (by water, salt ions, and any other solvent molecules) of the macromolecule is the Perrin translational frictional function, $P$ (see following equation). This degree of water association is termed the hydration of the solute, $\delta$, and is defined as the mass in grams of associated solvent per gram of anhydrous solute ${ }^{53}$.

$$
P=\frac{f}{f_{0}}\left(\frac{\bar{v}}{\bar{v}_{s}}\right)^{1 / 3}=\frac{f}{f_{0}}\left(\frac{\bar{v}}{\bar{v}+\delta \bar{v}_{H 2 O}}\right)^{1 / 3}
$$

where $f / f_{0}$ is the frictional ratio (which is the dimensionless ratio of the observed translational 
1 frictional coefficient $f$ to that of an equivalent spherical molecule of the same anhydrous mass and density

$\left.2 \mathrm{f}_{0}\right), \bar{v}$ is the partial specific volume $\left(\mathrm{cm}^{3} / \mathrm{g}\right)$ of the solute and $\bar{v}_{s}$ is the hydrated specific volume (the 3 volume occupied by the solute and associated solvent per unit mass of the anhydrous solute) and $\bar{v}_{H 2 O}=$ 4 specific volume of water given as:

$$
\bar{v}_{S}=\bar{v}+\delta \bar{v}_{H 2 O}
$$

If all excess friction is due to the hydration and the solute is a sphere, then $P$ is $1^{54}$ and the hydration can be calculated as:

$$
\delta=\frac{\left[\left(\frac{f}{f_{0}}\right)^{3}-1\right] \bar{v}}{\bar{v}_{H 2 O}}=\left[\left(\frac{f}{f_{0}}\right)^{3}-1\right] \bar{v} \rho_{H 2 O}
$$

Hydrated content analysis. The moles of water molecules per mole of $\mathrm{CaCO}_{3}$ was thus calculated according to the result of inductively coupled plasma atomic emission spectroscopy (ICP-AES), TG analysis and volumetric Karl Fischer titration measurement. Titration measurement was performed on a Karl Fischer moisture titrator (V10S, volumetric KF titrator, Mettler Toledo). The hydrated content was assigned to be the weight loss starting from the endothermic peak to $300{ }^{\circ} \mathrm{C}$, while the content of $\mathrm{Ca}^{2+}$ and $\mathrm{Gd}^{3+}$ ions were determined by ICP-AES. The molar ratio between water and $\mathrm{Ca}^{2+}$ ions in ACC can thus be calculated according to previous reports ${ }^{20,55,56}$.

Leakage studies of $\mathbf{G d}^{3+}$ ion. The detection of leakage of gadolinium ion was carried out using a method reported previously ${ }^{28,29,57}$. ACNC were dispersed in a series of simulative physiological environment including normal saline, human serum and human serum supplemented an additional phosphate concentration of $10 \mathrm{mmol} / \mathrm{L}$. ACNC was dispersed with a final concentration of $1 \mathrm{mmol}(\mathrm{Gd}) / \mathrm{L}$. The dialysis of ACNC was performed at $37{ }^{\circ} \mathrm{C}$ for 7 days using a dialysis bag (MWCO $1000 \mathrm{Da}$ ). The concentration of gadolinium in dialysates collected at different time points was measured by both 
1 Arsenazo III mediated chromogenic assay and ICP-AES. In the chromogenic assay, the collected 2 dialysates were mixed with Arsenazo III (0.05 mM) dissolved in chloroacetic acid-sodium hydroxide buffer solution ( $\mathrm{pH} 2.8$ ), and the absorption at $658 \mathrm{~nm}$ was detected. Normal saline and $\mathrm{GdCl}_{3}$ solution were used as negative and positive controls, respectively.

In vitro biocompatibility studies. Human umbilical vein endothelial cells (HUVEC) were cultured in Dulbecco's modified Eagle's medium. Cell viability was performed by standard MTT method. Briefly, cells were plated at the density of about $1 \times 10^{4}$ cells per well in 96-well plate, and incubated for $12 \mathrm{~h}$. Then DMEM culture medium with ACNC were added, and cells were exposed to ACNC at various concentrations for $24 \mathrm{~h}$. Then $10 \mu \mathrm{L}$ of MTT solution ( $5 \mathrm{mg} / \mathrm{mL}$ in PBS) was added to each well for additional $4 \mathrm{~h}$ incubation at $37^{\circ} \mathrm{C}$. After removing the medium, $150 \mu \mathrm{L}$ of DMSO was added to dissolve the formed formazan crystals in each well, and the optical density of the solution was measured at $570 \mathrm{~nm}$ using Microplate Reader (BioTek Instruments, USA). Human renal tubular epithelial (HK-2) cells were used for mitochondrial membrane potentials (MMP) assay, TdT-mediated dUTP Nick-End Labeling (TUNEL) assay and 5-Ethynyl-2'-deoxyuridine (EdU) assay. Mitochondrial membrane potential assay kit with J-aggregates (JC-1) (Beyotime, Shanghai, China), one step TUNEL apoptosis assay kit (Beyotime, Shanghai, China), and EdU cell proliferation kit with Alexa Fluor 555 (Beyotime, Shanghai, China) were employed.

Animal studies. Specific pathogen Free (SPF) BALB/c mice (male, 6 weeks), New Zealand rabbit (male, $2 \mathrm{~kg}$ ) and Beagle dog (male, $6 \mathrm{~kg}$ ) were purchased from Anhui Medical University. Animal studies were performed in accordance with the recommendations in the Guide for the Care and Use of Laboratory Animals of the National Institutes of Health. The in vivo compatibility studies and in vivo MRI studies were under protocols approved by the Institutional Animal Care and Use Committee (IACUC) of Anhui 
2 In vivo biocompatibility studies. ACNC normal saline solutions were intravenously injected in mice (via

3 tail vein) at a dosage of $5 \mathrm{mg} \mathrm{Gd} / \mathrm{kg}$ bodyweight and Beagle dog (via hindlimb vein) at a dosage of $9 \mathrm{mg}$

$4 \mathrm{Gd} / \mathrm{kg}$ bodyweight. For evaluation of blood index and biochemical index, mice were sacrificed by

5 anesthetics for 3 days and 3 weeks after intravenous injection, respectively. Blood of Beagle dogs were

6 collected every two weeks via hindlimb vein. Blood samples were collected by the anticoagulant blood

7 collection tubes and separating gel tubes. The examination of blood index and biochemical index were

8 carried out on Sysmex XE2100 and Vitros 5600, respectively.

9 In vivo metabolic study. ACNC normal saline solutions were intravenously injected into twenty mice 10 (male, $20 \mathrm{~g}$ ) at $3 \mathrm{mg} \mathrm{Gd} / \mathrm{kg}$ bodyweight $(0.5 \mathrm{mg} \mathrm{Gd} / \mathrm{mL}, 120 \mu \mathrm{L}$ ) via rapid manual injection for a simulated bolus injection study. These mice were randomly divided into four groups, and various organs were resected from the mice in each group 1, 7, 15 and 30 days after intravenous injection, respectively. The urine samples were collected at 12 hours after i.v. injection of ACNC on rat at $5 \mathrm{mg} \mathrm{Gd} / \mathrm{kg}$ bodyweight, followed by dialysis at $25{ }^{\circ} \mathrm{C}$. After 24 hours, the dialysates in dialysis bags (MWCO=1000 Da) were collected for further characterization.

MRI acquisition and analysis. ACNC and Gd-DTPA dispersed in normal saline at gradient concentrations in $5 \mathrm{~mL}$ microtubes were placed on a support immersed in $\mathrm{NiSO}_{4}$ solution. The concentrations of gadolinium were measured by ICP-AES. MR $T_{1}$ map were acquired using an inversion recovery sequence on a 3.0 Tesla MR scanner (Trio Tim, Siemens) equipped with a head coil. Imaging parameters were as follows: repetition time $(\mathrm{TR})=4000 \mathrm{~ms}$, echo time $(\mathrm{TE})=14 \mathrm{~ms}$, inversion time $(\mathrm{TI})$ from 25 to $3500 \mathrm{~ms}$ (TI included 25, 50, 75, 100, 150, 200, 250, 300, 350, 400, 500, 600, 800, 1000, 1500, $2000,2500,3000,3500 \mathrm{~ms})$, field of vision $(\mathrm{FOV})=220 \times 144 \mathrm{~mm}^{2}$. The longitudinal relaxivity $\left(r_{l}\right)$ was 
1 calculated as follow according to an equation reported previously with a concentration gradient from 0.025

2 to $0.4 \mathrm{mM}$ of gadolinium ion. The inverse of the relaxation time $\left(1 / T_{1}, \mathrm{~s}^{-1}\right)$ was plotted against the 3 concentration of gadolinium, and the slope of the plot was the relaxivity of the agent $\left(\mathrm{mM}^{-1} \cdot \mathrm{s}^{-1}\right)^{58,59}$. The 4 measurements on a low field MR scanner system were performed using a $0.5 \mathrm{~T}$ LF-NMR instrument at $5 \quad 32{ }^{\circ} \mathrm{C}$ provided by Suzhou Niumag Analytical Instrument Corporation.

6 In vivo MR angiography analysis. After complete intravenous anaesthesia, animals were fixed onto 7 different supports with suitable size. Then, ACNC dispersed in normal saline or Gd-DTPA diluted by 8 normal saline were injected intravenously. Rat was injected from caudal vein using an indwelling needle 9 at a dose of $2.5 \mathrm{mg} \mathrm{Gd} / \mathrm{kg}$. Bolus injection on rabbit and beagle dog were performed at a dose of $3 \mathrm{mg}$ FLASH 3D sequence was employed to collect angiographic data. The detailed parameters of MR angiography of rat were as follows: A knee coil was used. TR $=3.95 \mathrm{~ms}$, TE $=1.9 \mathrm{~ms}, \mathrm{FOV}: 210 \times 280$ $\mathrm{mm}^{2}$. The time of acquisition (TA) was 24.62 seconds. Flip angle (FA) was 20. Acquisition matrix was $288 \mathrm{p} * 512$. Acquisition number and number of averages were both 1 . The detailed parameters of MR angiography of rabbit were as follows: A local head and neck coil were used. $\mathrm{TR}=3.6 \mathrm{~ms}, \mathrm{TE}=1.65 \mathrm{~ms}$, FOV: $210 \times 280 \mathrm{~mm}^{2}$. The time of acquisition (TA) was 23.4 seconds. Flip angle (FA) was 18 . Acquisition matrix was $288 p * 512$. Acquisition number and number of averages were both 1 . The detailed parameters of MR angiography of beagle dog were as follows: A local head and neck coil, spine coil, and radiofrequency body transmit coil were used. TR $=3.19 \mathrm{~ms}, \mathrm{TE}=1.28 \mathrm{~ms}, \mathrm{FOV}: 240 \times 320 \mathrm{~mm}^{2}$. The time of acquisition (TA) was 20.74 seconds. Flip angle (FA) was 16. Acquisition matrix was 288p * 512 . Acquisition number and number of averages were both 1 . The images were processed firstly by Siemens Syngo MR workstation. 
2

(ROIs). One in the vessel of interest $\left(\mathrm{ROI}_{\mathrm{vessel}}\right)$ were measured by placing a signal at the same ROI in the same slice both in Gd-DTPA and ACNC group, which was recorded as the mean signal intensities of vessel $\left(\mathrm{S}_{\mathrm{vessel}}\right)$. One in the image background $\left(\mathrm{ROI}_{\text {background }}\right)$ was located in an artifact-free homogeneous area, which was defined as the background signal ( $\left.\mathrm{S}_{\text {background }}\right)^{31}$. SNR corresponds to the ratio between $\mathrm{S}_{\text {vessel }}$ and Sbackground $_{\text {as follow: }}$

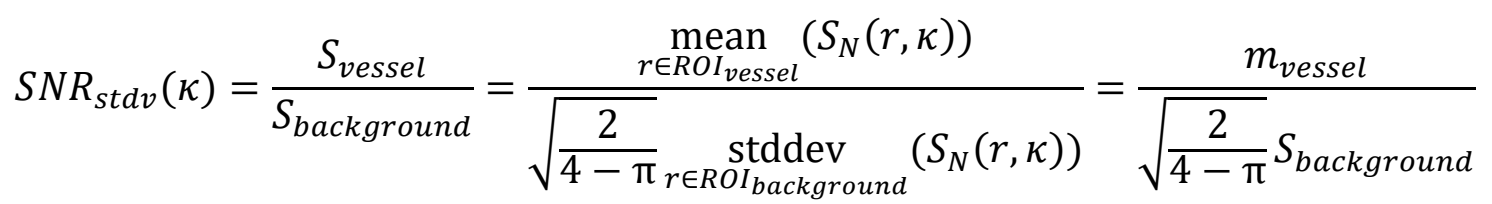

Three ROIs were selected in descending aorta, aortic arch and ascending aorta on rabbit, and four regions in brachiocephalic trunk artery, left subclavian artery, left common carotid artery and right branch of olfactory artery were measured on beagle dog, respectively.

Statistical analysis. All data were expressed as mean \pm S.E.M. and analyzed by independent samples ttests. A value of $\mathrm{P}<0.05$ was considered statistically difference. $\left({ }^{*} \mathrm{P}<0.05,{ }^{* *} \mathrm{P}<0.01,{ }^{* * *} \mathrm{P}<0.001\right)$.

Data availability. The data that support the findings of this study are available on request from the corresponding authors (Y.L., H.C. or S.-H.Y.).

\section{References}

1. De Yoreo, J. J., et al. Crystallization by particle attachment in synthetic, biogenic, and geologic environments. Science 349, 10 (2015).

2. Politi, Y., Arad, T., Klein, E., Weiner, S., Addadi, L. Sea urchin spine calcite forms via a transient amorphous calcium carbonate phase. Science 306, 1161-1164 (2004). 
3. $\mathrm{Xu}, \mathrm{Y}$. F., et al. Microscopic structure of the polymer-induced liquid precursor for calcium carbonate. Nat. Commun. 9, 12 (2018).

4. Addadi, L., Raz, S., Weiner, S. Taking advantage of disorder: Amorphous calcium carbonate and its roles in biomineralization. Adv. Mater. 15, 959-970 (2003).

5. Liu, Z. M., et al. Crosslinking ionic oligomers as conformable precursors to calcium carbonate. Nature 574, 394-398 (2019).

6. Gower, L. B. Biomimetic model systems for investigating the amorphous precursor pathway and its role in biomineralization. Chem. Rev. 108, 4551-4627 (2008).

7. Du, H. C., Amstad, E. Water: How does it influence the $\mathrm{CaCO}_{3}$ formation? Angew. Chem. Int. Edit. 59, 1798-1816 (2020).

8. Ihli, J., et al. Dehydration and crystallization of amorphous calcium carbonate in solution and in air. Nat. Commun. 5, 3169 (2014).

9. Alberic, M., et al. The crystallization of amorphous calcium carbonate is kinetically governed by ion impurities and water. Adv. Sci. 5, 9 (2018).

10. Farhadi-Khouzani, M., Chevrier, D. M., Zhang, P., Hedin, N., Gebauer, D. Water as the key to proto-aragonite amorphous $\mathrm{CaCO}_{3}$. Angew. Chem. Int. Edit. 55, 8117-8120 (2016).

11. Gebauer, D., et al. Proto-calcite and proto-vaterite in amorphous calcium carbonates. Angew. Chem. Int. Edit. 49, 8889-8891 (2010).

12. Zou, Z. Y., et al. A hydrated crystalline calcium carbonate phase: Calcium carbonate hemihydrate. Science 363, 396-400 (2019).

13. Smeets, P. J., Cho, K. R., Kempen, R. G., Sommerdijk, N. A., De Yoreo, J. J. Calcium carbonate nucleation driven by ion binding in a biomimetic matrix revealed by in situ electron microscopy. Nat. Mater. 14, 394-399 (2015).

14. Wahsner, J., Gale, E. M., Rodriguez-Rodriguez, A., Caravan, P. Chemistry of MRI contrast agents: current challenges and new frontiers. Chem. Rev. 119, 957-1057 (2019).

15. Dong, H., et al. Lanthanide nanoparticles: from design toward bioimaging and therapy. Chem. Rev. 115, 10725-10815 (2015).

16. Huang, H., Feng, W., Chen, Y., Shi, J. L. Inorganic nanoparticles in clinical trials and translations. Nano Today 35, 100972 (2020).

17. Loste, E., Wilson, R. M., Seshadri, R., Meldrum, F. C. The role of magnesium in stabilising 
amorphous calcium carbonate and controlling calcite morphologies. J. Cryst. Growth 254, 206$218(2003)$.

18. Shannon, R. D. Revised effective ionic radii and systematic studies of interatomic distances in halides and chalcogenides. Acta Crystallogr. A A32, 751-767 (1976).

19. Smith, D. W. Ionic hydration enthalpies. J. Chem. Educ. 54, 540-542 (1977).

20. Huang, S.-C., Naka, K., Chujo, Y. A carbonate controlled-addition method for amorphous calcium carbonate spheres stabilized by poly(acrylic acid)s. Langmuir 23, 12086-12095 (2007).

21. Ünaleroğlu, C., Zümreoğlu-Karan, B., Özcan, S., Firat, T. Characterization and magneticbehavior of cobalt(Ii) and gadolinium(Iii) polyacrylates. J. Appl. Polym. Sci. 56, 1239-1243 (1995).

22. Kellermeier, M., et al. Colloidal stabilization of calcium carbonate prenucleation clusters with silica. Adv. Funct. Mater. 22, 4301-4311 (2012).

23. Michel, F. M., et al. Structural characteristics of synthetic amorphous calcium carbonate. Chem. Mater. 20, 4720-4728 (2008).

24. Khouzani, M. F., et al. Disordered amorphous calcium carbonate from direct precipitation. Crystengcomm 17, 4842-4849 (2015).

25. Gebauer, D., Völkel, A., Cölfen, H. Stable prenucleation calcium carbonate clusters. Science 322, 1819-1822 (2008).

26. Laurent, S., Elst, L. V., Copoix, F., Muller, R. N. Stability of MRI paramagnetic contrast media A proton relaxometric protocol for transmetallation assessment. Invest. Radiol. 36, 115-122 (2001).

27. Laurent, S., Vander Elst, L., Henoumont, C., Muller, R. N. How to measure the transmetallation of a gadolinium complex. Contrast Media Mol. Imaging 5, 305-308 (2010).

28. Kumar, R., Nyk, M., Ohulchanskyy, T. Y., Flask, C. A., Prasad, P. N. Combined optical and MR bioimaging using rare earth ion doped $\mathrm{NaYF}_{4}$ nanocrystals. Adv. Funct. Mater. 19, 853-859 (2009).

29. Wang, L. J., et al. A Gd-doped Mg-Al-LDH/Au nanocomposite for CT/MR bimodal imagings and simultaneous drug delivery. Biomaterials 34, 3390-3401 (2013).

30. Frenzel, T., Lengsfeld, P., Schirmer, H., Hutter, J., Weinmann, H. J. Stability of gadolinium-based magnetic resonance imaging contrast agents in human serum at $37^{\circ} \mathrm{C}$. Invest. Radiol. $43,817-828$ (2008). 
31. Dietrich, O., Raya, J. G., Reeder, S. B., Reiser, M. F., Schoenberg, S. O. Measurement of signalto-noise ratios in MR images: Influence of multichannel coils, parallel imaging, and reconstruction filters. J. Magn. Reson. Imaging 26, 375-385 (2007).

32. Park, S. M., Aalipour, A., Vermesh, O., Yu, J. H., Gambhir, S. S. Towards clinically translatable in vivo nanodiagnostics. Nat. Rev. Mater. 2, 19 (2017).

33. Lee, H., Shin, T. H., Cheon, J., Weissleder, R. Recent developments in magnetic diagnostic systems. Chem. Rev. 115, 10690-10724 (2015).

34. Chen, H. M., Zhang, W. Z., Zhu, G. Z., Xie, J., Chen, X. Y. Rethinking cancer nanotheranostics. Nat. Rev. Mater. 2, 18 (2017).

35. Min, Y. Z., Caster, J. M., Eblan, M. J., Wang, A. Z. Clinical translation of nanomedicine. Chem. Rev. 115, 11147-11190 (2015).

36. Du, B. J., Yu, M. X., Zheng, J. Transport and interactions of nanoparticles in the kidneys. Nat. Rev. Mater. 3, 358-374 (2018).

37. Du, B. J., et al. Glomerular barrier behaves as an atomically precise bandpass filter in a subnanometre regime. Nat. Nanotechnol. 12, 1096-1102 (2017).

38. Jin, Y. D., Jia, C. X., Huang, S. W., O'Donnell, M., Gao, X. H. Multifunctional nanoparticles as coupled contrast agents. Nat. Commun. 1, 8 (2010).

39. Raz, S., Hamilton, P. C., Wilt, F. H., Weiner, S., Addadi, L. The transient phase of amorphous calcium carbonate in sea urchin larval spicules: The involvement of proteins and magnesium ions in its formation and stabilization. Adv. Funct. Mater. 13, 480-486 (2003).

40. Rodriguez-Navarro, C., Kudlacz, K., Cizer, O., Ruiz-Agudo, E. Formation of amorphous calcium carbonate and its transformation into mesostructured calcite. Crystengcomm 17, 58-72 (2015).

41. Glatter, O. Evaluation of small-angle scattering data from lamellar and cylindrical particles by the indirect transformation method. J. Appl. Crystallogr. 13, 577-584 (1980).

42. Ravel, B., Newville, M. ATHENA, ARTEMIS, HEPHAESTUS: data analysis for X-ray absorption spectroscopy using IFEFFIT. J. Synchrot. Radiat. 12, 537-541 (2005).

43. Stern, E. A. Number of relevant independent points in X-ray-absorption fine-structure spectra. Phys. Rev. B, Condens. Matter 48, 9825-9827 (1993).

44. Booth, C. H., Hu, Y. J. Confirmation of standard error analysis techniques applied to EXAFS using simulations. In: DiCicco A, Filipponi A (eds). 14th International Conference on X-Ray Absorption Fine Structure, vol. 190. Iop Publishing Ltd: Bristol, 2009. 
45. https://www.nanolytics-instruments.de/interference_optics_aida.

46. Schuck, P. P. S. v. c. http://analyticalultracentrifugation.com/download.htm.

47. Schuck, P., Rossmanith, P. Determination of the sedimentation coefficient distribution by leastsquares boundary modeling. Biopolymers 54, 328-341 (2000).

48. Carney, R. P., et al. Determination of nanoparticle size distribution together with density or molecular weight by 2D analytical ultracentrifugation. Nat. Commun. 2, 335 (2011).

49. Brown, P. H., Schuck, P. Macromolecular size-and-shape distributions by sedimentation velocity analytical ultracentrifugation. Biophy. J. 90, 4651-4661 (2006).

50. Institute of Materials, École Polytechnique Fédérale de Lausanne (EPFL), Station 12, 1015 Lausanne, Switzerland E-mail: quy.ong@epfl.ch.

51. Demeler, B. U. v., release 2783. . A Comprehensive Data Analysis Software Package for Analytical Ultracentrifugation Experiments. The University of Lethbridge, Department of Chemistry and Biochemistry.

52. Brookes, E., Cao, W. M., Demeler, B. A two-dimensional spectrum analysis for sedimentation velocity experiments of mixtures with heterogeneity in molecular weight and shape. Eur. Biophys. J. Biophys. Lett. 39, 405-414 (2010).

53. Harding, S. E. Frictional coefficient, ratio. In book: T. E. Creighton, . Encyclopedia of Molecular Biology., (J. Wiley \& Sons, New York, 1999). pp.1942.

54. C. R. Cantor, P. R. S. Biophysical Chemistry. Part II. Techniques for the study of biological structure and function, (W. H. Freeman, 1980) pp. 1561-1586.

55. Ihli, J., Kulak, A. N., Meldrum, F. C. Freeze-drying yields stable and pure amorphous calcium carbonate (ACC). Chem. Commun. 49, 3134-3136 (2013).

56. Radha, A. V., Forbes, T. Z., Killian, C. E., Gilbert, P., Navrotsky, A. Transformation and crystallization energetics of synthetic and biogenic amorphous calcium carbonate. Proc. Natl. Acad. Sci. U. S. A. 107, 16438-16443 (2010).

57. Hvattum, E., Normann, P. T., Jamieson, G. C., Lai, J. J., Skotland, T. Detection and quantitation of gadolinium chelates in human serum and urine by high-performance liquid chromatography and post-column derivatization of gadolinium with Arsenazo III. J. Pharmaceut. Biomed. 13, 927-932 (1995).

58. Fram, E. K., et al. Rapid calculation of $\mathrm{T}_{1}$ using variable flip angle gradient refocused imaging. Magn. Reson. Imaging 5, 201-208 (1987). 
159 Kim, B. H., et al. Large-scale synthesis of uniform and extremely small-sized iron oxide nanoparticles for high-resolution $\mathrm{T}_{1}$ magnetic resonance imaging contrast agents. J. Am. Chem. Soc. 133, 12624-12631 (2011). 


\section{Acknowledgements}

2 This work was supported by the National Natural Science Foundation of China (Grants 51732011, 21431006, 21761132008, 51972090, 21501039, 51702309, 81801831), the Foundation for Innovative

4 Research Groups of the National Natural Science Foundation of China (Grant 21521001), Key Research

5 Program of Frontier Sciences, CAS (Grant QYZDJ-SSW-SLH036), the National Basic Research Program 6 of China (Grant 2014CB931800), the Users with Excellence and Scientific Research Grant of Hefei 7 Science Center of CAS (2015HSC-UE007), the Fundamental Research Funds for the Central Universities 8 (WK9110000062, JZ2018HGPA0269, WK2060190056), the China Postdoctoral Science Foundation 9 (2015M570540), the Natural Science Foundation of Anhui Province (1708085ME114), the Major/Innovative Program of Development Foundation of Hefei Center for Physical Science and Technology (2016FXZY005). The authors would like to thank Mei Sun, Yan-Wei Ding, Cheng-Min Wang, and Technology of China, Hao Ding in Suzhou Niumag Analytical Instrument Corporation, He Chen, Li Zhang and Hui Wang in The First Affiliated Hospital of Anhui Medical University, Duo An in Cornell University, and Ye-Ping Li from Anton Paar China for useful assistance of this manuscript, and Luca Olivi, thanks the particle analysis center of the SFB 1214 for the AUC equipment.

\section{Author Contributions}


1 S.H.Y. and Y.L. conceived the idea, designed the experiments and supervised the research, L.D., C.S., Y.L.,

2 Y.Z., L.B.M., D.G., H.C., R.R., Y.D.W. and F.L. carried out the synthetic experiment and analysis. D.G., 3 J.A., H.C. and L.D. worked on the EXAFS measurement and analysis. L.D., Y.Z., H.L.G., Z.P., H.Q.W., $4 \quad$ X.Y. and F.L. processed the biocompatible evaluation. L.D., Y.J.X., Y.D.W., Y.L., H.L.G., Z.P., X.Y., F.L. 5 and C.S. worked on the animal experiments, L.D., Y.J.X., Y.L., F.L. and C.S. investigated the MRI 6 performance, L.D., Y.J.X., C.S., D.G., Y.L., H.C. and S.H.Y. wrote the paper, all authors discussed the 7 results and commented on the manuscript.

\section{$9 \quad$ Additional information}

10 Supplementary Information accompanies this paper at http://www.nature.com/naturecommunications

11 Competing interests: The authors declare no competing interests.

Reprints and

permissions

information

is

available

online

at

http://npg.nature.com/reprintsandpermissions/

Publisher's note: Springer Nature remains neutral with regard to jurisdictional claims in published maps and institutional affiliations. 


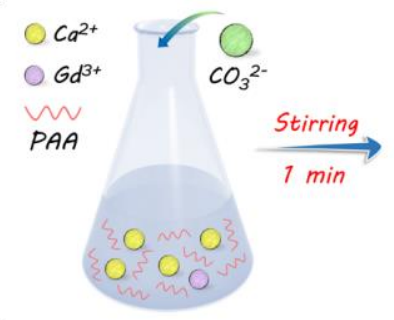

d
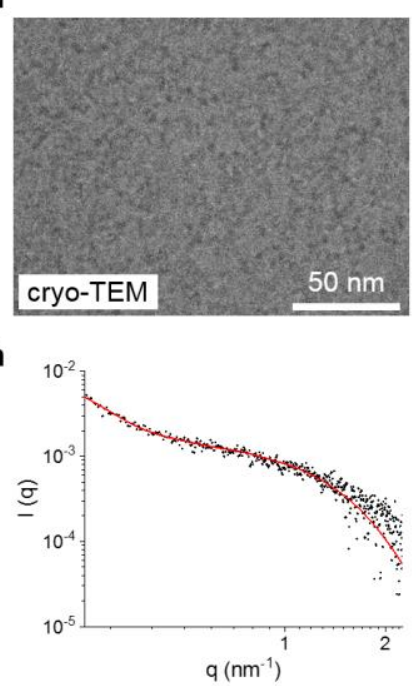

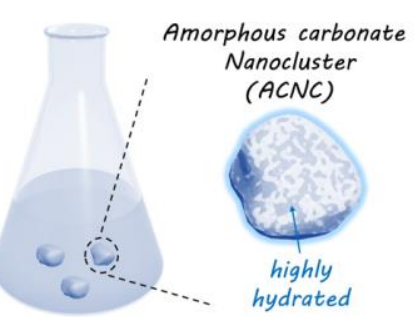

e
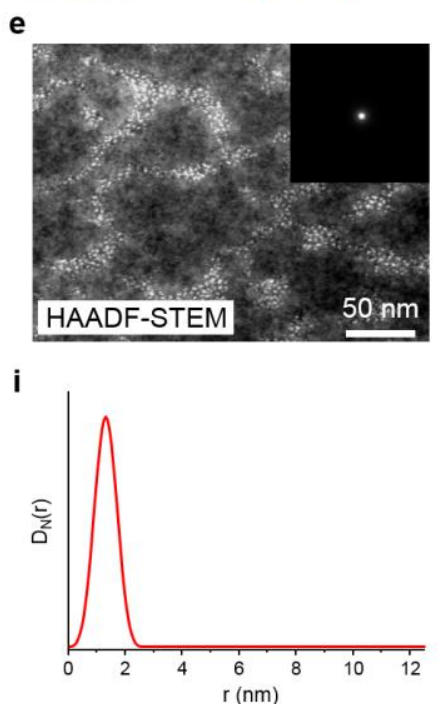

b
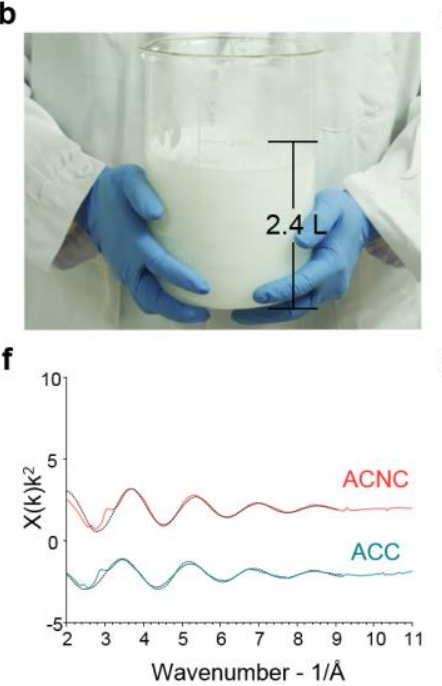

j

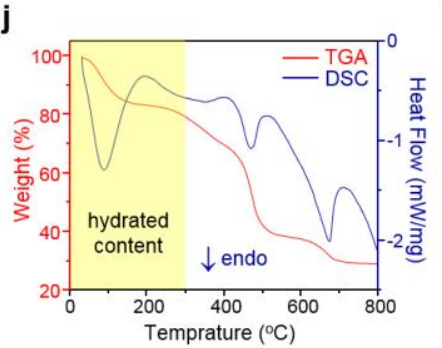

c

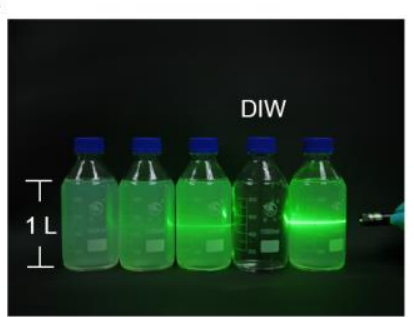

g

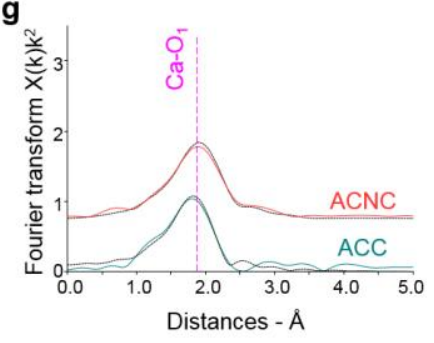

k

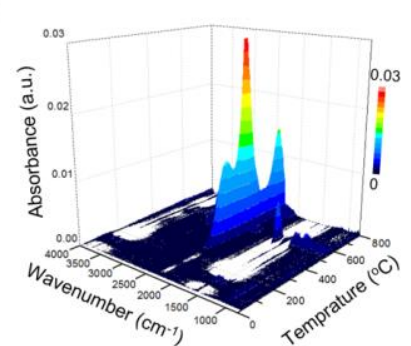

Figure 1 | Characterization of amorphous carbonate nanocluster (ACNC). a Scheme of the synthesis process of ACNC. b Digital image of the large-scale prepared ACNC with the total volume over 2 litres.

c Digital image of Tyndall effect of four litres of ACNC dispersed in normal saline, and one bottle of deionized water was inserted (second from right). d cryo-TEM and e HAADF-STEM image of ACNC dispersed in normal saline solution. The inset in $\mathbf{e}$, shows SAED of ACNC. $\mathbf{f} \mathrm{k}^{2}$-weighted EXAFS and $\mathbf{g}$ $\mathrm{k}^{2}$-weighted Fourier transform of the EXAFS for the ACNC and ACC standard. Dotted black lines are their best fits. $\mathbf{h}$ SAXS patterns of ACNC dispersed in normal saline solution. Red solid line fit sphere particles. i Distance distribution received by SAXS of ACNC dispersed in normal saline solution. j TGA of ACNC powder under an $\mathrm{N}_{2}$ atmosphere with a heating rate of $10^{\circ} \mathrm{C} \mathrm{min}^{-1}$. $\mathbf{k}$ TG-FTIR spectra of ACNC powder under an $\mathrm{N}_{2}$ atmosphere with a heating rate of $10{ }^{\circ} \mathrm{C} \mathrm{min}^{-1}$. 
a

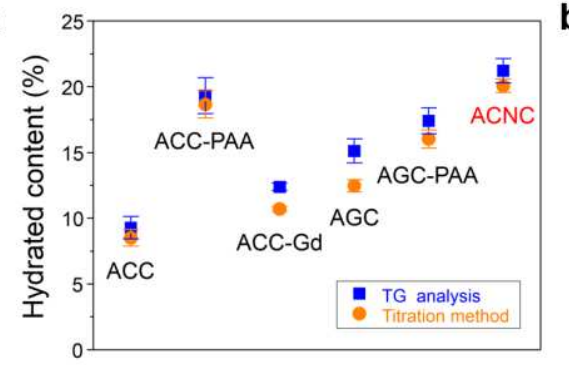

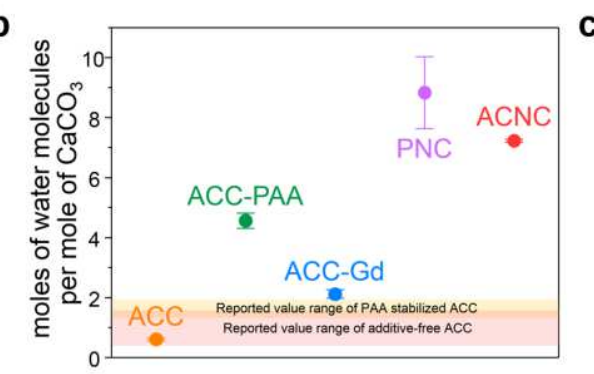

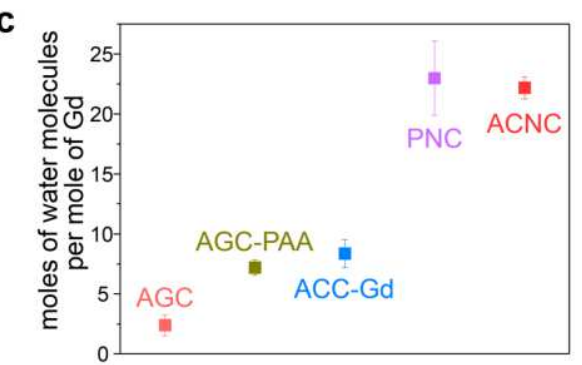

2 Figure 2 High water content of ACNC. a The water contents of ACC, ACC-PAA, ACC-Gd, AGC,

3 AGC-PAA and ACNC according to thermogravimetric (TG) analysis and volumetric Karl Fischer titration

4 measurements. b Molar ratio of water molecules per mole of $\mathrm{CaCO}_{3}$ in ACC, ACC-PAA, ACC-Gd and

5 ACNC $(\mathrm{n}=3)$ in comparison with the reported value ranges of additive-free ACC (pink region) and PAA-

6 stabilized ACC (orange region). c Molar ratio of water molecules per mole of Gd in AGC, AGC-PAA,

7 ACC-Gd and ACNC $(\mathrm{n}=3)$.

8

9 
a

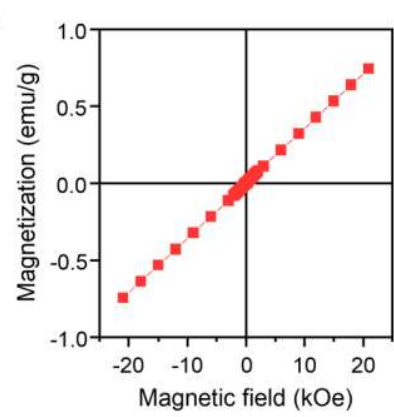

d

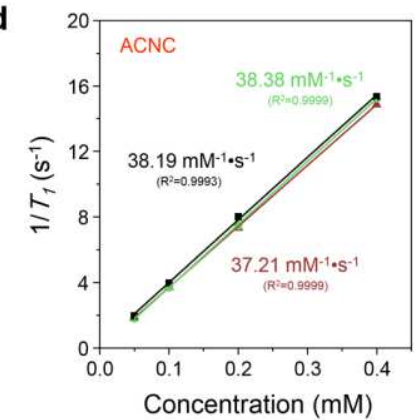

b

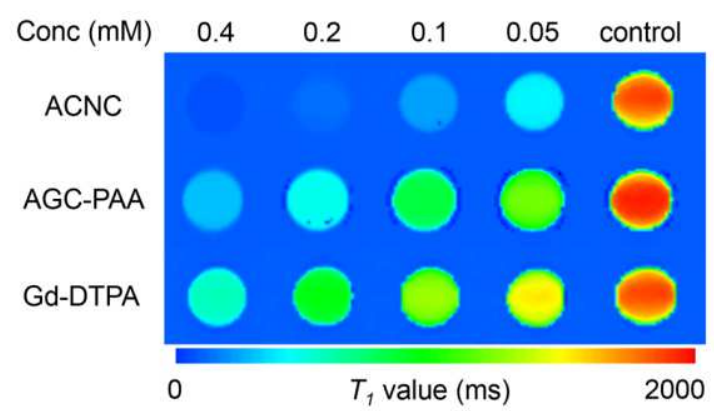

e
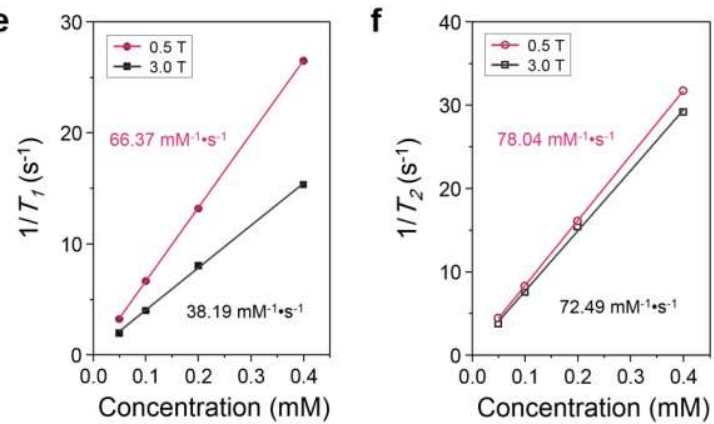

c

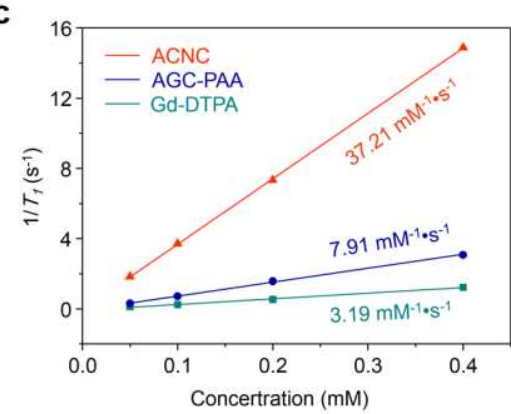

g

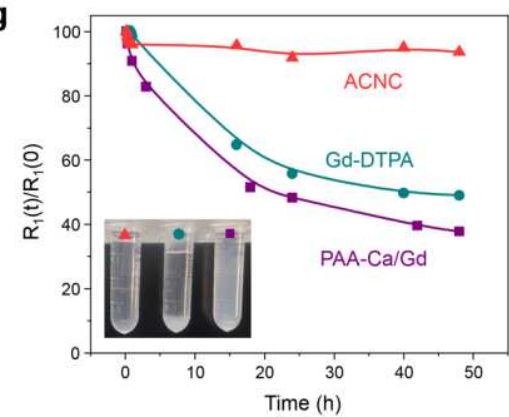

Figure 3 MRI performance of ACNC. a M-H curve of ACNC at room temperature. b-c $T_{1}$ map and

$31 / T_{1}$ versus Gd concentration curve of ACNC $\left(\mathrm{R}^{2}=0.9999\right)$, AGC-PAA $\left(\mathrm{R}^{2}=0.9987\right)$ and Gd-DTPA $\left(\mathrm{R}^{2}\right.$

$4=0.9966)$ under $3.0 \mathrm{~T}$. d $1 / T_{l}$ versus Gd concentration curve of ACNC under $3.0 \mathrm{~T}(\mathrm{n}=3)$. e $1 / T_{l}$ versus

5 Gd concentration curve of ACNC under $3.0 \mathrm{~T}\left(\mathrm{R}^{2}=0.9993\right)$ and $0.5 \mathrm{~T}\left(\mathrm{R}^{2}=0.9999\right)$. f $1 / T_{2}$ versus $\mathrm{Gd}$

6 concentration curve of ACNC under 3.0 T $\left(\mathrm{R}^{2}=0.9991\right)$ and $0.5 \mathrm{~T}\left(\mathrm{R}^{2}=0.9999\right)$. $\mathrm{g}$ Evolution of relative

$7 \quad \mathrm{R}_{1}$ values from the zero time to each time $\left(\mathrm{R}_{1}(\mathrm{t}) / \mathrm{R}_{1}(0)\right)$ for ACNC, Gd-DTPA and PAA-Ca/Gd. 

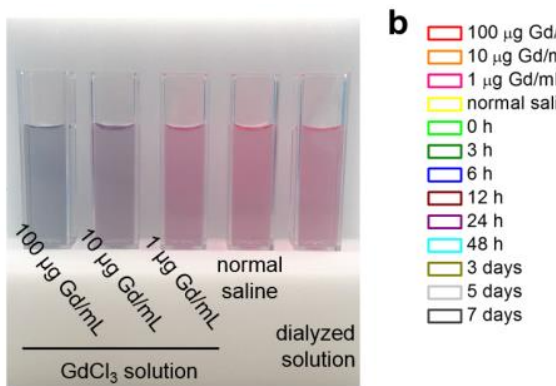

$\mathrm{GdCl}_{3}$ solution

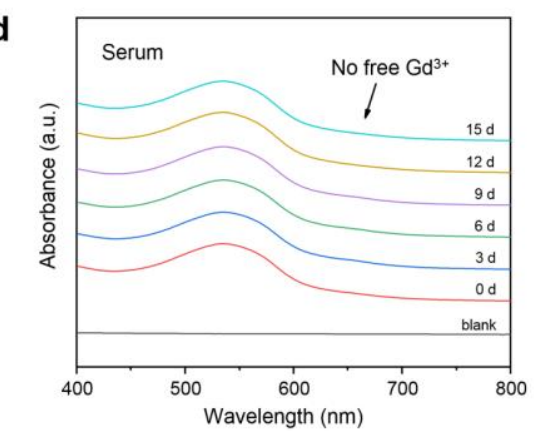

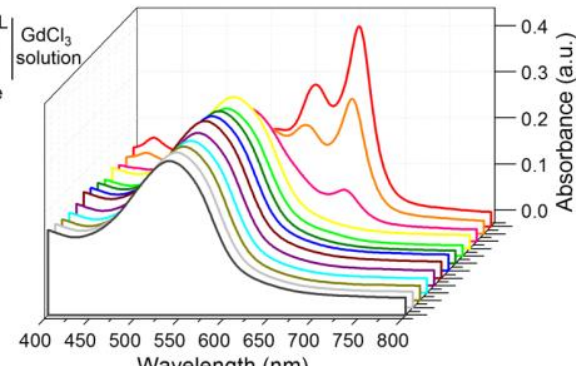

Wavelength $(\mathrm{nm})$
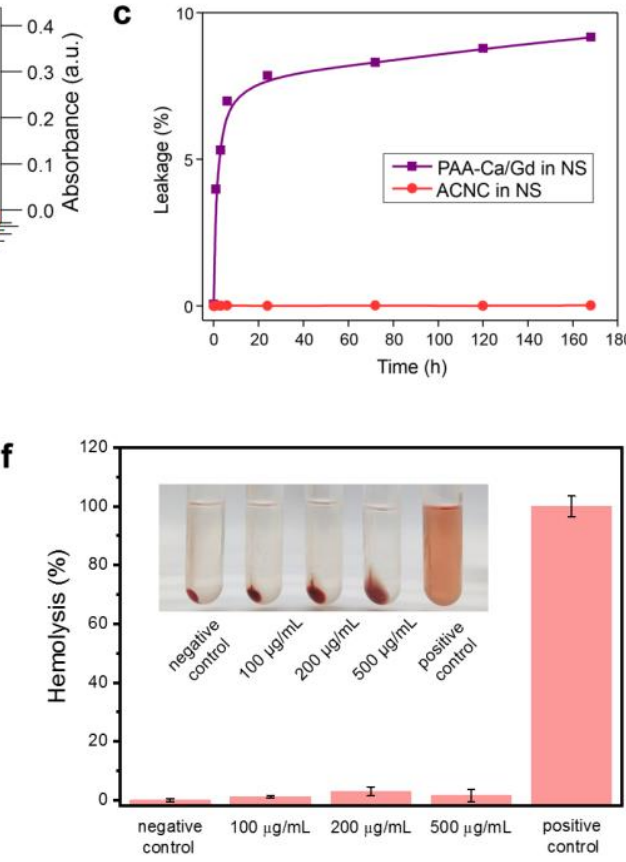

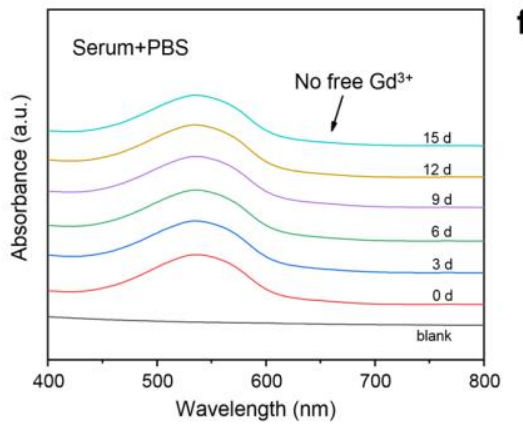

Figure $4 \mid$ Stability study. a Photos of the mixtures of Arsenazo III aqueous solution with dialyzed solutions of ACNC, normal saline (NS, served as negative control) and gadolinium chloride aqueous solution at varying concentrations (1, 10 and $100 \mu \mathrm{g} \mathrm{Gd} / \mathrm{mL}$ ) (served as positive controls), respectively. b Absorption spectra of arsenazo III-dialysate mixtures, and dialysates were collected at varying time points in 7days to monitor the leakage of gadolinium from ACNC. No detectable leakage of gadolinium ion was monitored compared with the negative (NS) and positive (gadolinium chloride aqueous solution) controls. c Quantitative analysis and comparison of the leakage of free gadolinium ion from $\mathrm{ACNC}$ and PAA-Ca/Gd in NS by means of ICP-AES. PAA-Ca/Gd chelate showed poor inertness in NS, and approximately $9 \%$ gadolinium was leaked from the chelate after 7 days. In contrary, few free gadolinium ion was detected in the dialysates of ACNC. d-e Absorption spectra of arsenazo III mixture with dialyzed solutions collected at different time points from $\mathbf{d}$ human serum with dispersed ACNC and e human serum with dispersed ACNC and supplemented additional phosphate. f Blood compatibility evaluation of ACNC at varying gadolinium concentration. 

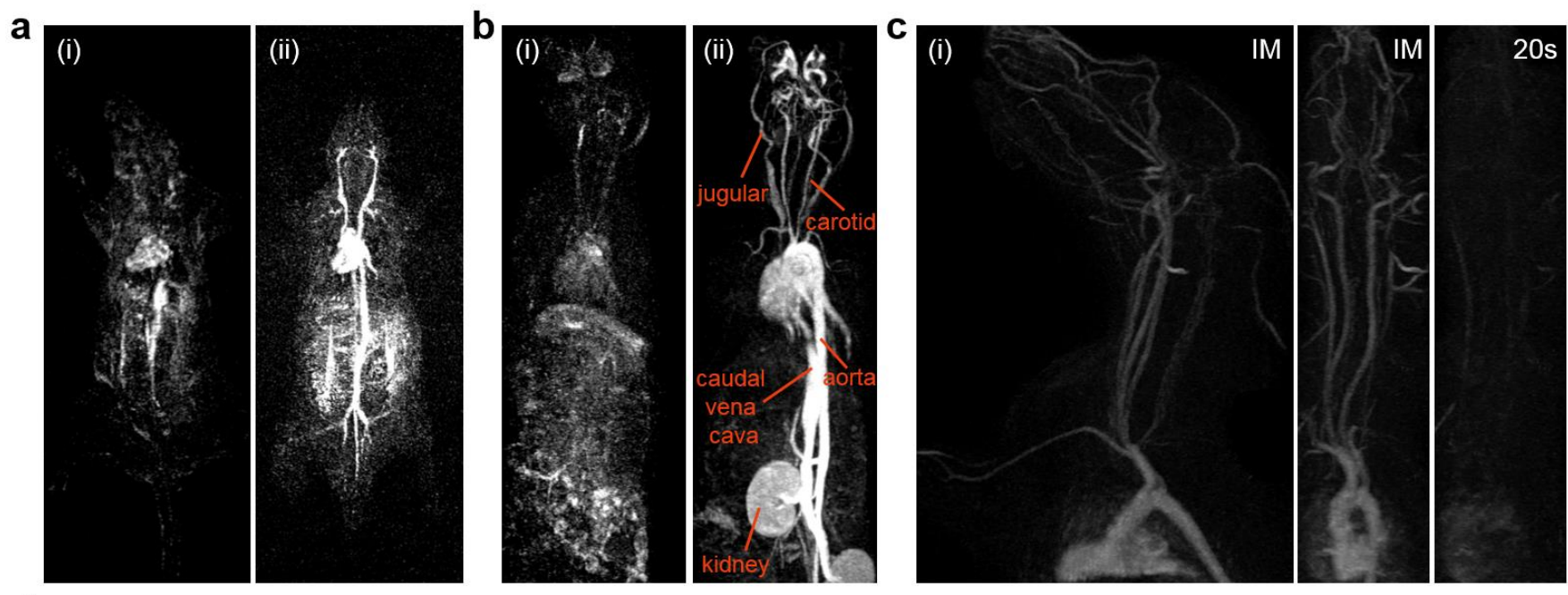

d
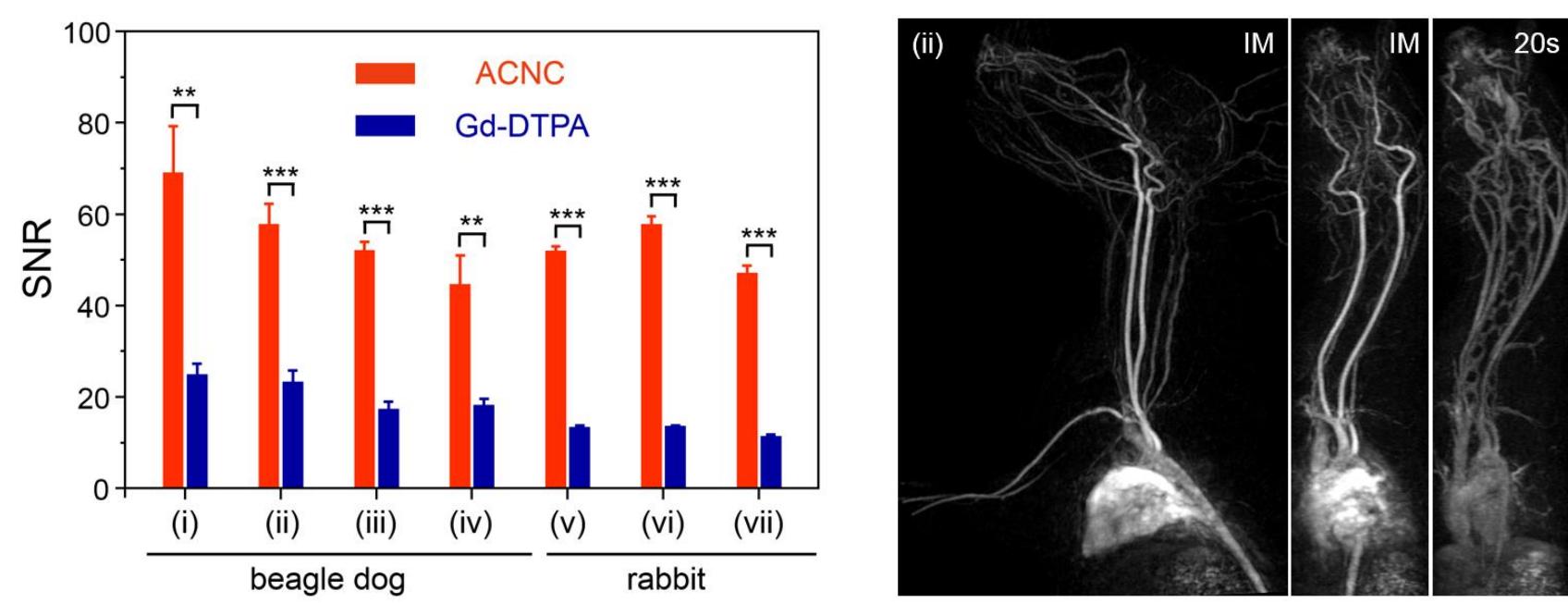

2 Figure $5 \mid$ In vivo MR angiography. a-b Contrast enhanced MR angiography (MRA) images of the whole

3 body on a rat and $\mathbf{b}$ rabbit immediately after the bolus injection of (i) Gd-DTPA and (ii) ACNC. c MRA

4 images of upper body on beagle dog at immediately (IM) and 20 seconds after the bolus injection of (i)

5 Gd-DTPA and (ii) ACNC, respectively. d Quantitative measurement of SNR of ROIs in (i) brachiocephalic

6 trunk artery, (ii) left subclavian artery, (iii) left common carotid artery, (iv) right branch of olfactory artery

7 of beagle dog, and that in (v) descending aorta, (vi) aortic arch, (vii) ascending aorta of rabbit, respectively.

8 The time for quantitative measurement of SNR of ROIs was in the "IM" period. (** $\mathrm{P}<0.01, * * * \mathrm{P}<0.001)$. 
a

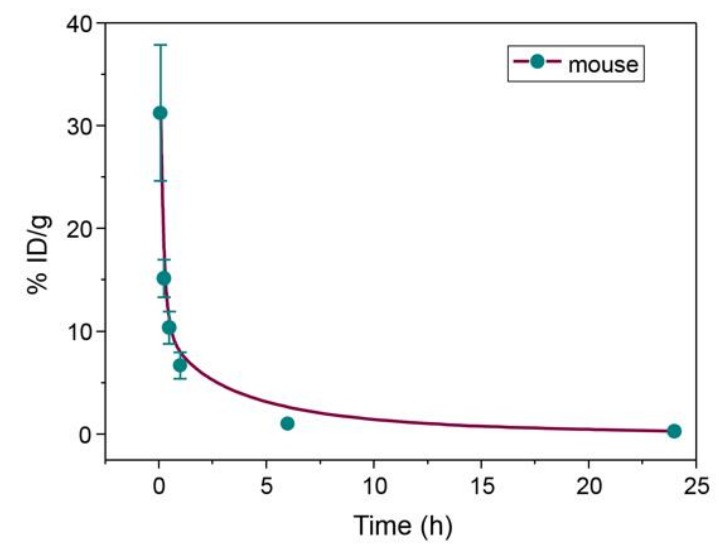

C

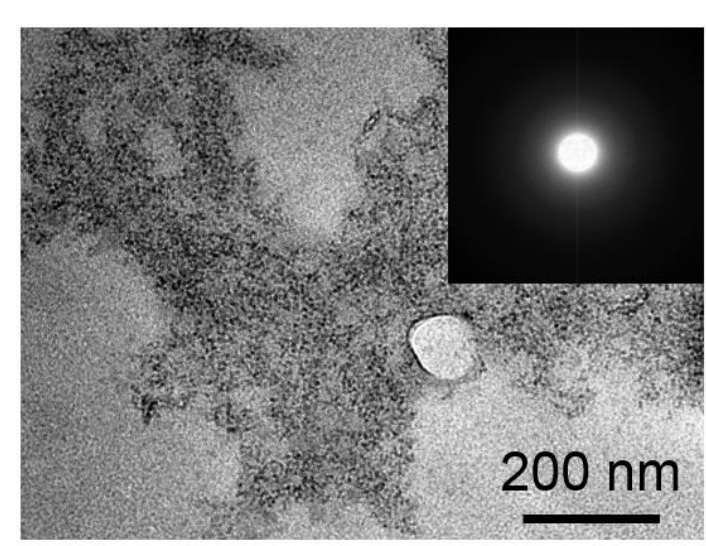

b

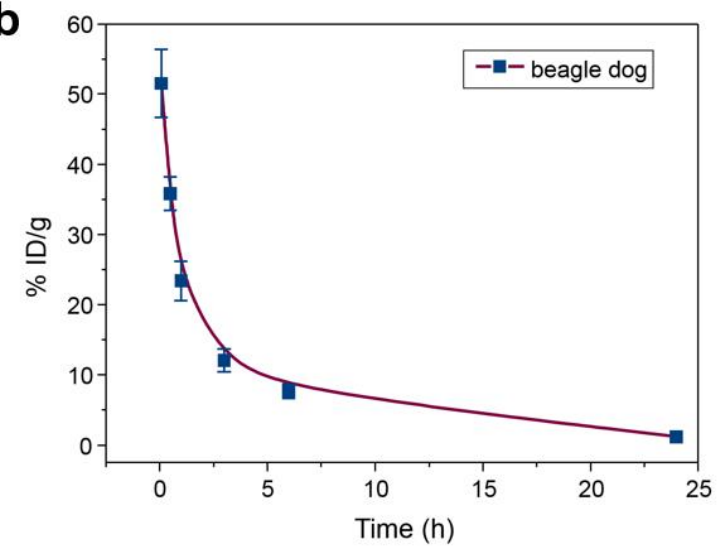

d

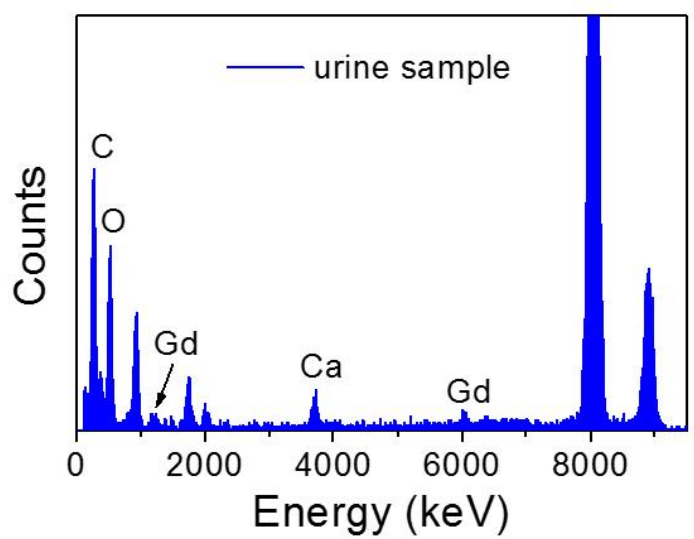

e
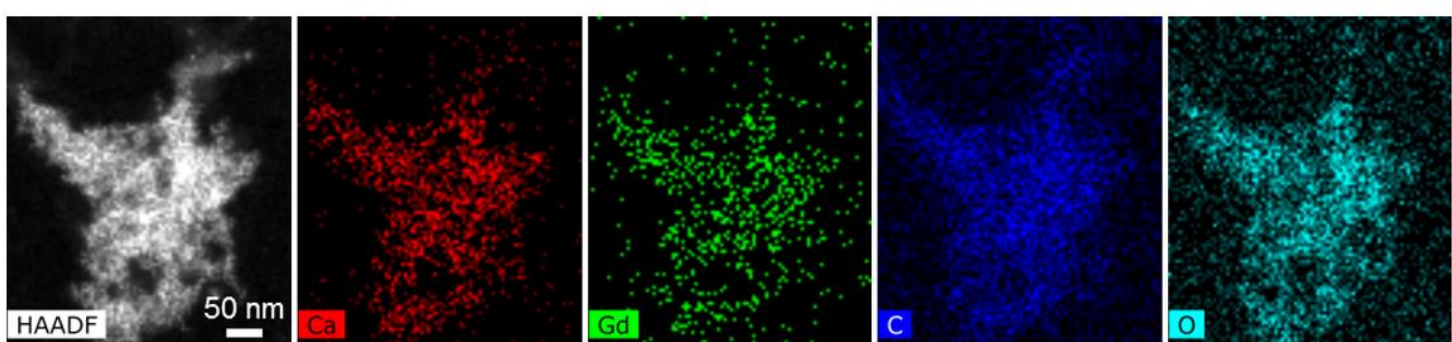

Figure 6 | Metabolism of ACNC. a-b The time dependent distribution of ACNC in plasma of a mice and

3 b beagle dogs within 24 hours. c TEM, $\mathbf{d}$ EDS results and e HAADF-STEM image and EDS mapping of

4 ACNC observed in rat urine collected 12 hours post-injection. Inset of $\mathbf{c}$ is relative SAED pattern. 


\section{Supplementary Files}

This is a list of supplementary files associated with this preprint. Click to download.

- SupplementaryinformationACNCNatCommun.docx 